10.2478/gb-2020-0006

sciendo

\title{
Dichten ums Überleben auf Gratwanderung zwischen Ost und West. Die Rolle der Geschichte(n) in Jan Koneffkes Roman Die sieben Leben des Felix Kannmacher
}

\section{Ana KARLSTEDT}

Lekt. Dr., Universität Bukarest; E-Mail: ana.karlstedt@1ls.unibuc.ro

MOTTO: Heute kommt es mir vor, als sei meine Erinnerung eine von diesen erfundenen Geschichten - eine Geschichte aus fremdem Land und fremder Zeit.(Koneffke 2011: S. 40)

\begin{abstract}
Looking back, my memories seem like distant, made-up stories. These words of the main character are to be found all over Jan Koneffkes novel. A foreigner on the run, Felix Kannmacher is forced to tell stories in order to survive. He is at the mercy of a teenager who is avid for constantly new and different bedtime stories. Felix gives in, as he has no other choice but to be a slaveto this child. The following article analyses the fine line between the stories Felix invents for Virginia and two narratological aspects: the actual plot of the novel on the one hand, and the actual course of history, on the other. The entire novel is built on stories-within-stories that twist and turn the course of Story and History alike. Because each one of us writes their own (hi)story.
\end{abstract}

Keywords: Possible Worlds Theory; Counterfactual / Alternate History; Story versus History

Wie ein Leitmotiv wiederholen sich im gesamten Buch die obigen Worte. Denn in seinem zwischen Orient und Okzident pendelnden historischen Roman schafft Jan Koneffke eine verführende Mischung aus Geschichten (Stories) erzählen und 
Geschichte (History) erzählen. Wie ein Antiheld, wie ein regelrechter Pícaro schildert Felix Kannmacher in Ich-Formseine mannigfaltigen Schicksale und Abenteuer, seine meist verbotenen, unerlaubten, gefährlichen Erlebnisse aus der Perspektive desjenigen, dessen Existenz an einem dünnen Faden hängt.

Getarnt durch eine neue, doch prekäre Identität, überlebt der vor den Nazis flüchtende Deutsche Felix nur, weil er für die adoleszente Tochter seines (scheinbaren ${ }^{1}$ ) rumänischen Retters Geschichten erfinden muss, um sie gnädig zu stimmen. Eine männliche Scheherezade im Kampf ums Überleben steht im Fokus dieser Arbeit. Denn Geschichte schreibt jeder anders (und jeder neu). Vor allem dann, wenn es ums Überleben geht, wenn der Akt des Geschichtenerzählens überlebenswichtig wird, und, auch in Anlehnung an Tausendundeine Nacht, kann manden Text als Gratwanderung zwischen Ost und West betrachten. Dies, dank der interkulturellen Beziehungen zwischen dem okzidentalisch anmutenden Protagonisten und den auf ihn fremd und orientalisch wirkenden rumänischen Einheimischen.

Mitte der Dreißiger Jahre wird also der gegen seinen Willen zum Geschichtenerfinder gewordenen Flüchtling Felix Kannmacher vom Lügenbaron, unwahre Geschichten über sich selbst verbreitenden Pianisten Victor Marcu² aus Nazideutschland ge-

${ }^{1}$ Es ist derselbe Marcu, der ihn, Felix, ohne zu zucken später im Roman aus dem Nest der zweiköpfigen, aus ihm und Virginia bestehenden Familie aus Eifersucht und Neid hinausschmeißt.

2 Es gehört zum Charme des Romans dass Victor Marcu, das perfekte Beispiel der Wendung fromragstoriches, selbst ein Geschichtenerzähler ist, da er seine beschämende Herkunft vertuschen möchte. Sehr gut ist Marcu mit dem Baron von Münchhausen vergleichbar. Hier Felix “ feine Beschreibung seines Retters: ,Seine Erinnerungen kamen nicht in Fluß, was mit den falschen Geschichten zusammenhing, die er bereits (...) in Umlauf gebracht hatte. Um vor seinen Kommilitonen zu bestehen, die aus besseren Hauptstadtfamilien stammten, gab er sich als Sproß eines Rittermeisters aus, den eine feindliche Kugel erwischt hatte, als Sohn beßarabischer Kaufleute oder als Nachkomme eines 
rettet. Marcus scheinbar altruistische Geste entpuppt sich aber mehr und mehr als Ausbeutung. Felix spürt von Beginn an das Joch des rumänischen Retters, die kurze Hundeleine, an der er von Virginia herumgeführt wird, die Kluft zwischen ihm und Marcus Freunden.

Felix bekommt demnach zu Beginn des Romans eine neue Identität und eine neue, für ihn ungewöhnliche Aufgabe. Er wird nämlich, ob er es will oder nicht, zur Kinderfrau von Marcus Tochter Virginia, bei der er sich nur als Geschichtenerfinder beliebt machen kann. Felix schöpft, in den neun Geschichten, die das Buch beleben und die Lektüre um so reizender machen, aus seiner Vergangenheit in Deutschland sowie aus der Gegenwart in Rumänien die verschiedensten Ideen für Gutenachtgeschichten. Denn alte, weltbekannte Geschichten möchte Virginia nicht haben.

Genau hier knüpft der hiesige Beitrag an. Thematisiert wird hier nämlich die Rolle dieser kontrafaktischen Geschichten für den Romanplot, für die Figuren, die in ihnen vorkommen - sowohl historische, als auch von Jan Koneffke erfundene Charaktere:

Meine Aufgabe war es, ein freches Biest zu unterhalten. Mir blieb keine andere Wahl. Ich mußte mir neue Geschichten ausdenken. Ich war erpreßbar, und meine Erpresserin war ein verzogenes Kind. Eine geschichtsvergessene Blage. Mir blieb keine andere Wahl. Ich mußte mir neue Geschichten ausdenken (...).(Koneffke 2011: S. 17)

Das verwöhnte, Felix schikanierende Problemkind Virginia, gibt sich mit herkömmlichen Märchen also nicht zufrieden. Sie durschaut jeden Versuch von Felix, ihr bereits Erzähltes wieder aufzutischen. Felix hat keine andere Wahl als sich den Kopf zu

verarmten Bojarengeschlechts aus dem tiefsten Moldawien. Vor Journalisten und Kritikern hatte er andere Geschichten erfunden. Bald war er ein Findlingskind, das man im Bauch eines griechischen Dampfers entdeckt hatte (oder in einem Weidenkorb aus hoher See). (Koneffke 2011: S. 109-110) 
zerbrechen, neue Geschichten zu erfinden, um die wilde und ungestüme Virginia zu zähmen:

Und was ist mit meiner Geschichte? Dauernd verlangte Virginia Geschichten von mir aus dem düsteren Land an der Ostsee. Leider fielen mir keine mehr ein. Sie kannte bereits alle Schauerlegenden und Sagen, die mir aus der Kindheit vertraut waren. Mit Wiederholungen abspeisen ließ sie sich nicht, Wiederholungen konnten sie fuchsteufelswild machen. Und sie merkte im Handumdrehen, wenn ich es wagte, einen pommerschen Schwank wiederholen $\mathrm{zu}$ wollen. (Koneffke 2011: S. 16)

Unter kontrafaktischer Geschichte versteht man eine ungewöhnliche Wendung im Lauf der überlieferten Geschichte -nämlich so, wie die historisch belegte Geschichte hätte sein können. Was Jan Koneffke durch seinen Protagonisten schafft ist es, den Weg faktischer, überlieferter Geschichte zu verändern. In diesem Prozess wird Geschichte umgeformt und Geschichte selbst, sowie Geschichtsschreibung entpuppen sich als Konstrukt: „Historyitselfis a textualconstruct.“ (Herman et. al. 2008: S. 86)

Ausgangspunkt für diesen Sachverhalt ist ,das Konzept der möglichen Welten, die Auffassung von der Wirklichkeit als modalem System (...), das aus einer Vielzahl von Welten besteht: einer tatsächlichen Welt (actualworld) und möglichen alternativen Welten (possibleworlds).“ (Nünning 2008: S. 584) Hier bekommen fiktionale Texte folgende Rolle zugeteilt: die als „semiotische Mechanismen für die Konstruktion alternativer Welten“ (ebd.). Das bedeutet, dass Autoren die Wirklichkeit nicht bloß nachahmen, sondern durch hinzugedichtete parallele Welten ersetzen. Folgende ausgewählte theoretische Aspekte aus der so genannten Possible Worlds Theory (weiter unten PWT) sind für die praktische Untersuchung des Romans relevant:

Im Rahmen der $P W T$ werden daher ganz unterschiedliche Fragestellungen untersucht:(a) die Frage nach der Bestimmbarkeit des Wahrheitswertes von Sachverhalten in fiktionalen Texten; (b)der Grad nach Autonomie bzw. Abhängigkeit fiktionaler Welten im 
Verhältnis zur außertextuellen Wirklichkeit; (c) das Problem der ontologischen Unvollständigkeit fiktionaler Entitäten; (...) (f) das Zusammenspiel von tatsächlichen (auf Handlungsebene verwirklichten) und virtuellen (von den Figuren erhofften oder geplanten) Ereignissen in einem Plot; (...) (h) die Frage nach der Beziehung zwischen real existierenden Individuen und ihren fiktionalen Ebenbildern (...) (Nünning 2008: S. 584)

Der hiesige Beitrag geht also auf die unterschiedliche, variierende Rezeption und Interpretation des literarischen Textes ein. Untersucht werden die Bezüge zwischen den vorbestimmten Leseerwartungen, der History und dem eigentlichen Roman geschehen. Diese Erwartungen formen, modellieren das Lektüreerlebnis. Der Beitrag geht auch der Frage nach, in wie fern die im Roman enthaltenen Geschichten auch autonom, außerhalb der eigentlichen Romanhandlung ihre Gültigkeit bewahren. Und dies tun sie auch. Das Spannende an der Untersuchung bleibt, beide Optionen beim Lesen offen zu halten: Dass einerseits die Geschichten eigenständig funktionieren könnten, andererseits aber stark mit dem Lauf der faktischen und fiktiver Geschichte miteinander verwoben sind. Und diese enge Beziehung besteht sowohl auf der Ebene: erfundene Geschichte versus Story, als auch auf der Ebene: erfundene Geschichte versus faktische History.

In der Erzähltheorie geht man also von der Prämisse aus, dass Dinge in der Welt auch anders sein könnten, als sie wirklich sind. Felix versüßt seine prekäre, düstere Existenz als Flüchtling durchs Geschichtenerzählen. ${ }^{3}$ Er imaginiert alternative, glückliche Szenarien für sich selbst und für seine malbeliebte, mal stiefmütterliche neue Heimat, Rumänien. Der tatsächlichen Welt stellt er, wie verdrehte Spiegelbilder, mögliche alternative

3 Allmählich wird das Geschichtenerzählen für Felix, über die Pflicht und Last hinaus, auch zum Genuss. Er beginnt, seine frei erfundenen Geschichten zu mögen, zu kultivieren, zu verbessern. Immer hat er Notizheft und Stift parat, um sich neue Einfälle zu notieren. 
Welten entgegen. Er macht parallele Wirklichkeiten vorstellbar und zugänglich. Manchmal gehen seine Geschichten in Richtung Fantastik. Die wissenschaftliche Frage, die sich hier stellt ist die nach dem Wahrheitswert von Sachverhalten in fiktionalen Texten. Problematisch wird die Glaubwürdigkeit des IchErzählers Felix - zumal, wie bereits erwähnt, die Handlung von dem Selbstzweifel Felix‘ gegenüber der Richtigkeit seiner Erinnerungen überschattet wird.

Kontrafaktische Geschichtserzählung, kontrafaktisches Denken haben als Grundlage die Intention, Geschichte neu zu erdenken. Es ist ein Spiel mit der History innerhalb der Stories, bei dem oft Humor, Ironie, Sarkasmus oder Zynismus präsent sind. Der Umgang ist also experimentell und spielerisch. Es entstehen durch den unzuverlässigen Erzähler Felix Kannmacher so genannte ungeschehene oder alternative Geschichten. Also gehört es sich für den Leser, den Text zu hinterfragen bzw. auf Spurensuche zu gehen, um herauszufinden, was Jan Koneffke hinter den vermeintlich frei erfundenen und frei funktionierenden Geschichten kaschiert.

Was steckt also hinter den Kulissen, wozu sind die erfundenen Geschichten da, außer um Virginia bei Laune zu halten? Ja, auf den ersten Blick richten sich die Stories an das heranwachsende, verzogene, von Geschichtenbesessene Mädchen. Blickt man aber hinter diese Fassade, so stellt man fest, dass der Hintergrund ein ganz anderer sein kann. Als volens nolens Historiograf spielt Felix Kannmacher mit dem Lauf der eigentlichen Geschichte. Er dreht historische Fakten um und verändert ihren Rahmen, ihre Wirkung, ihren Ausgang. Und das vor allem im Kontext einschneidender historischer Umbrüche. Er lässt auf diese Weise veränderte Geschichtsmodelle sichtbar werden.

Felix bringt Unerwartetes, Unwahrscheinliches ans Licht. Er rückt Neues in den Vordergrund und schafft somit ein neues Geschichtsverständnis, das überlieferte Daten in Frage stellt und gleichzeitig nicht nur Vergangenes, sondern auch Gegenwärti- 
ges und Zukünftiges mit verändert. Seine Darstellung ist verfremdend. So geht beispielsweise der Zweite Weltkrieg gut aus, wie sich im Laufe des Beitrags zeigen wird. Dabei sind die fiktionalen Geschichtsalternativen ein beinahe operativer Eingriff in den Wahrheitsgehalt bzw. in die Faktizität der Geschichte. Das Verhältnis zwischen historischer Realität und davon abweichender literarischer Fantasie bringt den Leser auf Reflexion, auf neue Gedanken. Die Geschichte bekommt so neue Gesichter - sie verwandelt sich in einen Probelauf historischer Möglichkeiten.

Welche Rolle haben, also, die erfundenen Geschichten in der Romanwelt? Sie appellieren auf spielerische Weise an das Vorwissen des Lesers, der plötzlich mit einer komplett neuen Art der Geschichtsüberlieferung konfrontiert wird. Im Geiste konstruiert der Leser diese neuen historischen Universen mit. Sie bewegen sich im Bereich des Was wäre wenn...? Oder, nach dem englischsprachigen Modell, stellen die Virginia erzählten Geschichten eine Welt des Whatif...? des couldhave ... shouldhave ... wouldhave.

Eine andere Frage, der dieser Beitrag nachgeht, ist, die nach dem Grad der Autonomie bzw. Abhängigkeit fiktionaler Welten im Verhältnis zur außertextuellen Wirklichkeit. Eine erste Deutungsmöglichkeit ist die, der Autonomie der Stories. Das heißt, dass die von Felix für Virginia erfundenen Geschichten auch für sich stehen könnten - als Elemente der Kinder-und Jugendliteratur. Die zweite Deutungsmöglichkeit hingegen setzt eine Abhängigkeit Story-History voraus.

Sehr interessant ist genau das Zusammenspiel von tatsächlichen und virtuellen Ereignissen in der Handlung des Romans. Es passieren historische Ereignisse auf der Handlungsebene, die Felix in den Geschichten für Virginia, anders aussehen und ausgehen lässt. Es stellt sich hier die Frage nach dem Wirklichkeitsbezug fiktionaler Texte: Koneffke spielt, durch seine Hauptfigur, mit der kulturellen Überlieferung historischer Ereignisse und dreht sie um. 
Ausgehend von der Auffassung, dass die Wirklichkeit, die wir zu sehen glauben, immer ein Konstrukt ist, umso mehr in einem literarischen, fiktionalen Werk, untersucht der vorliegende Beitrag das Verhältnis zwischen Story und History in diesem Buch. Besonders ausgeprägt ist dieses Verhältnis gerade bei historischen Romanen: Die sieben Leben des Felix Kannmacher ist, über einen Abenteuer-, Schelmen- und Bildungsroman, vor allem ein historischer Roman.

Das Verhältnis zwischen Fiktionalität und Historizität, Story und History, muss vom Leser sorgfältig, peuà peu, entdeckt werden. Vom Leser zu finden sind die Nahtstellen, welche Ähnlichkeiten, Differenzen, Unstimmigkeiten, Unbekanntes aufweisen. Leser erkennen diese Fiktion-Wirklichkeitsbezüge dadurch, dass sie diese mit ihrer Erfahrung und mit ihren Geschichtskenntnissen verknüpfen und vergleichen. Es gibt keine objektive Wirklichkeit, sondern eine subjektive Selektion der Wirklichkeit. Sie wird in diesem Roman durch Felix, den voreingenommenen, vorbelasteten Beobachter, konstruiert bzw. erfunden, es ist also seine subjektive, konstruierte Wirklichkeit.

Die Geschichten, die sich Felix für Virginia ausdenkt, erinnern an Buch- und Filmgeschichten wie Roberto Benignis $L a$ vita è bella oder Radu Mihaileanus Train de vie oder Jurek Beckers Jakob der Lügner. In der italienischen Tragikomödie befinden sich die Protagonisten in einem Konzentrationslager. Hier erfindet ein Vater, für seinen Sohn, ein neues Szenario für ihre Bleibe und für den gesamten Kontext, in dem sich beide befinden. Alles, was die Bösen, Kapos und Lagerkommandant wörtlich sagen, wird vom Vater komplett anders Train de vie, auch eine filmische Produktion zum Thema Drittes Reich und Zweiter Weltkrieg endet durch zwei verschiedene Schlussszenen: Eine, in der die History ihren bekannten Lauf nimmt - alle Lagerinsassen sterben - und eine, in der es ein Happy-End gibt. So, als würde der Regisseur es den Zuschauern überlassen, welches Ende sie präferieren. Schließlich ist im Roman von 
Jurek Becker (und übrigens auch in dessen Verfilmung mit Robin Williams als Titel- und Hauptfigur) die Rede davon, dass (wiederum auch in einem jüdischen Ghetto) ein junger Mann sich dafür entscheidet, seinen Mitmenschen das Leid zu lindern - durch erfundene, positive Nachrichten, die er in einem nicht vorhanden Radio angeblich hört. Gemeinsamer Nenner dieser Beispiele bleibt die Verschönerung der History.

Genau das erreicht auch Felix Kannmacher mit seinen Geschichten: Von den insgesamt neun Geschichten, die sich mit dem Plot des Romans sowie mit der Geschichte Rumäniens verbinden, haben es viele damit zu tun, dass die Geschichte, die History, anders dargestellt wird als wir sie aus Überlieferungen kennen. Was wäre gewesen, wenn...? So wird einer auf Handlungsebene ermordeten Figur das zurückgegeben, was sie zu Lebzeiten verloren hat, eine Art Wiedergutmachung. Es ist, also, eine kompensatorische Funktion für die Story, in diesem Fall:

Und es schloss sich sein Loch in der Kehle. Und alle Verletzungen verheilten im Nu, Stiche in Lunge und Leber, zerschmetterte Knochen, zerfetztes Gewebe. Und seine glasigen Augen belebten sich. Und Slumowitz ruckte und ruckelte in seinem Maßschneideranzug, der nicht mehr zerrissen war. Und er hob seine Pranken, die protzig beringt waren, an jedem Finger ein anderer Edelstein, um sein Sultansgesicht zu betasten.

(Koneffke 2011: S. 282)

In der Geschichte vom Spiel um Tod und Leben geht es darum, dass Felix'Beschützer, der jüdische Kasinobesitzer Aristide Slumowitz, von den Legionären ermordet und in einem Schlachthaus zur Schau gestellt wird. Was Felix in seiner kontrafaktischen Geschichte für ihn schafft, das ist, ihn regelrecht von den Toten zu den Lebenden wiederzubefördern. Er gibt seinem ehemaligen Vorgesetzten die Chance, die ihm im richtigen Leben verweigert wurde. Unverkennbar ist Jan Koneffkes Humor auch dadurch, dass der Jude Slumowitz sich in sein Sultans gesicht 
fasst. Auch hier eine schöne Melange aus Vorstellungen von Ost und West.

Eine zweite Geschichte, die in diesem Beitrag exemplarisch untersucht werden soll, ist die Kleine Geschichte von Großmut und Milde. Was wäre gewesen, wenn es ein positives, alternatives Ende des Zweiten Weltkrieges gegeben hätte? Ganz im Sinne Quentin Tarantinos Inglorious Basterds geht die Geschichte / History glimpflich aus - es ist eine kompensatorische Funktion für die History:

Und als endlich der Frieden ausbrach, wollte alle Welt freiwillig auf die im Krieg (...) besetzten Gebiete verzichten. Großherzig bot Josef Stalin den Polen einen Teil der Sowjetunion an. Und der kleinlaute Hitler trat, ohne zu zaudern, den Osten des Deutschen Reiches an seine Nachbarn ab. (...) Dieser Virus der Großherzigkeit steckte alle an. (Koneffke 2011: S. 334)

Felix macht also einen gefährlichen Spagat zwischen Ost und West. Zwischen Story und History. Er pendelt zwischen Extremen. Die Geschichten für Virginia funktionieren einerseits ohne Story und auch ohne History, sind also autonom. Andererseits stehen sie in einem stringenten Kausalitätsverhältnis. Das Geschichtenerzählen kann sehr wohl als Überlebensstrategie gesehen werden - somit ist und bleibt Felix eine männliche Scheherezade. Geschichten und Phantasie sind als Zufluchtsort, als Ventil zu verstehen. Sie stellen Felix ' metaphorische Wirklichkeitsflucht dar.

Wie in einem Märchen muss der Held (hier: Felix) viele gefährliche Situationen durchleben, aus denen er heil davonkommt. Um der Realität zu entfliehen, die nicht so ist, wie er sie sich wünscht, appelliert Felix an die Kraft der Worte und Erzählungen. Das große Abenteuer von Felix Kannmacher beginnt in den imaginierten Geschichten, die er sich für Virginia ausdenken muss. Wie das funktioniert, hat der hiesige Beitrag bewiesen: Felix ist eine männliche Scheherezade die das Geschichtenerzählen einerseits für sich selbst und sein Überleben, andererseits 
um die Welt von dem teuflischen Mechanismus der History zu retten, erzählt und erzählt und erzählt. Ioana Pârvulescu schreibt darüber: „De remarcat sunt și fermecătoarele povești inventate (Felix e und povestaș de geniu, o Șeherezadă care-și apără viața prin harul lui special de a fabrica povești), cu care personajul corectează istoria și destinul.” (Pârvulescu 2018: S. 291)

Schlussfolgernd kann man festhalten, dass die Hauptfigur in Jan Koneffkes (auch Kinderbuchautor und passionierter Geschichtensammler ${ }^{4}$ ) Roman mit Geschichten gegen den Lauf der Geschichte gewappnet ist:

Warum erfindet man eine Geschichte? (...) Um keine Geisel der Zeit mehr zu sein. Wer Geschichten erfindet, kann sie verlangsamen oder beschleunigen, dehnen oder stauchen. Er kann sie umkehren, anhalten, stillstellen, wenn er sich von einer Erinnerung nicht trennen will, einem Gesicht, einem Menschen. Nichts ist unwiderruflich und nichts, was sich in der Vergangenheit abspielte, notwendig. (Die) Geschichte beginnt an dem fernen Punkt in der Vergangenheit, als nichts entschieden war und das Leben noch einen ganz anderen Verlauf nehmen konnte. (Koneffke 2011: S. 329)

\section{LITERATUR}

\section{Primärliteratur}

\section{Bücher}

Becker, Jurek: Jakob der Lügner. Berlin Weimar 1969.

Koneffke, Jan: Die sieben Leben des Felix Kannmacher. Köln 2011.

Pârvulescu, Ioana:Dialoguri secrete. București 2018.

Weil, Gustav (Übers.): Tausendundeine Nacht. Wiesbaden 1982.

${ }^{4}$ Vgl. Jan Koneffke im Interview - Das Dings an sich -in der Berliner Zeitung vom 22. August 2015. 


\section{Filme}

Benigni, Roberto (Regie): La vita è bella 1997.

Kassovitz, Peter (Regie): Jacob the Liar. 1999.

Mihăileanu, Radu (Regie): Train de vie. 1998.

Tarantino, Quentin (Regie): Inglorious Basterds. 2012.

\section{Sekundärliteratur}

Herman, Jahn, Ryan (Hrsg.): Routledge Encyclopedia of Narrative Theory. London New York 2005.

Nünning, Ansgar (Hrsg.): Metzler Lexikon Literatur- und Kulturtheorie. 4. Auflage. Stuttgart Weimar 2008.

Internetquellen (zuletzt eingesehen am 01.05.2020)

Jan Koneffke im Interview: Das Dings an sich 7.9.2015 - 21:01, Berliner Zeitung, Interview: Sabine Vogel: https://www. berliner-zeitung.de/kultur-vergnuegen/ jan-koneffeke-im-interview-das-dings-an-sich-li.31715 